\title{
COMBINED EFFECTS OF DEPLETED URANIUM AND IONISING RADIATION ON ZEBRAFISH EMBRYOS
}

\author{
C. Y. P. Ng 1 , S. Pereira ${ }^{2}$, S. H. Cheng ${ }^{3,4}$, C. Adam-Guillermin ${ }^{2}$, J. Garnier-Laplace ${ }^{5}$ and K. N. Yu ${ }^{1,4, *}$ \\ ${ }^{1}$ Department of Physics and Materials Science, City University of Hong Kong, Hong Kong \\ ${ }^{2}$ Institut de Radioprotection et de Sûreté Nucléaire (IRSN), PRP-ENV/SERIS/LECO, Cadarache, \\ St Paul Lez Durance 13115, France \\ ${ }^{3}$ Department of Biomedical Sciences, City University of Hong Kong, Hong Kong \\ ${ }^{4}$ State Key Laboratory in Marine Pollution, City University of Hong Kong, Hong Kong \\ ${ }^{5}$ Institut de Radioprotection et de Sûreté Nucléaire (IRSN), PRP-ENV/SERIS, Cadarache, \\ St Paul Lez Durance 13115, France \\ *Corresponding author: peter.yu@cityu.edu.hk
}

\begin{abstract}
In the environment, living organisms are exposed to a mixture of stressors, and the combined effects are deemed as multiple stressor effects. In the present work, the authors studied the multiple stressor effect in embryos of the zebrafish (Danio rerio) from simultaneous exposure to alpha particles and depleted uranium (DU) through quantification of apoptotic signals at $24 \mathrm{~h}$ post-fertilisation (hpf) revealed by vital dye acridine orange staining. In each set of experiments, dechorionated zebrafish embryos were divided into 4 groups, each having 10 embryos: Group $(C)$ in which the embryos did not receive any further treatment; Group (IU) in which the embryos received an alpha-particle dose of $0.44 \mathrm{mGy}$ at $5 \mathrm{hpf}$ and were then exposed to $100 \mu \mathrm{g} \mathrm{l} \mathrm{I}^{-1}$ of $\mathrm{DU}$ from 5 to $6 \mathrm{hpf}$; Group (I) in which the embryos received an alpha-particle dose of $0.44 \mathrm{mGy}$ at 5 hpf and Group (U) in which the dechorionated embryos were exposed to $100 \mu \mathrm{g} \mathrm{I}^{-1}$ of DU from 5 to $6 \mathrm{hpf}$. The authors confirmed that an alpha-particle dose of $0.44 \mathrm{mGy}$ and a DU exposure for $1 \mathrm{~h}$ separately led to hormetic and toxic effects assessed by counting apoptotic signals, respectively, in the zebrafish. Interestingly, the combined exposure led to an effect more toxic than that caused by the DU exposure alone, so effectively DU changed the beneficial effect (hormesis) brought about by alpha-particle irradiation into an apparently toxic effect. This could be explained in terms of the promotion of early death of cells predisposed to spontaneous transformation by the small alpha-particle dose (i.e. hormetic effect) and the postponement of cell death upon DU exposure.
\end{abstract}

\section{INTRODUCTION}

In realistic situations, living organisms are exposed to a mixture of environmental stressors (e.g. ionising radiations, heavy metals etc.), and the combined effects are deemed as multiple stressor effects. Multiple stressor effects might not be simply the sum of effects from individual stressors ${ }^{(1,2)}$ and had been usually considered to include additive, synergistic or antagonistic effects. However, most of these combined effects were defined for toxic effects of the individual stressors. Now that hormesis has been widely accepted as a universal phenomenon for different stressors ${ }^{(3,4)}$; it would be interesting to explore the multiple stressor effect when one or more stressors are in the hormetic zone instead of the toxic zone.

In the present paper, the authors studied the multiple stressor effect of depleted uranium (DU) in the toxic zone and alpha-particle irradiation in the hormetic zone. Such a combination has real-life relevance. For example, in areas close to nuclear fuel cycle facilities, both uranium and alpha-emitting radionuclides are expected to be present in the environment. As the second example, DU armament started to be extensively used in the Gulf War. Some wounded personnels with DU fragments retained in their bodies ${ }^{(5)}$ would receive relatively high DU exposures while at the same time relatively low alpha-irradiation doses from the natural environment such as those from radon progeny.

In the present work, the multiple stressor effect was studied using embryos of the zebrafish, Danio rerio. The zebrafish and human genomes share considerable homology, including conservation of most DNA repairrelated genes ${ }^{(6)}$. Radiation effects on zebrafish embryos have been studied ${ }^{(7-11)}$, including the hormetic effect ${ }^{(8)}$. The authors' group previously studied the multiple stressor effect of alpha particles and cadmium using zebrafish embryos $^{(12-14)}$.

\section{MATERIALS AND METHODS}

\section{Zebrafish embryos}

Adult zebrafish (Danio rerio) were kept in fish tanks at $28{ }^{\circ} \mathrm{C}$. A $14 / 10 \mathrm{~h}$ light-dark cycle was adopted to maintain a good production of embryos. When the 14-h photoperiod began, a specially designed plastic collector was lowered onto the bottom of each tank to collect the embryos for 15 to $30 \mathrm{~min}$ to ensure the synchronisation of developmental stages of the embryos. The collected embryos were then transferred to a $28{ }^{\circ} \mathrm{C}$ incubator. At $4 \mathrm{~h}$ post-fertilisation (hpf), 
healthy developing embryos at the sphere stage of the blastula period were selected under a stereomicroscope and transferred into a Petri dish lined with agarose gel on the bottom and filled with $\mathrm{E} 3$ medium $(5 \mathrm{~mm} \mathrm{NaCl}$, $0.17 \mathrm{~mm} \mathrm{KCl}, 0.33 \mathrm{~mm} \mathrm{CaCl}_{2}, 0.33 \mathrm{~mm} \mathrm{MgSO}_{4}$ and $0.1 \%$ methylene blue) to facilitate dechorionation.

\section{Grouping of zebrafish embryos}

In each set of experiments, the dechorionated zebrafish embryos were divided into four groups, each having 10 embryos, and accommodated in four separate Petri dishes lined with agarose on the bottom. The four groups were referred to as:

(1) Control group (C): in which the dechorionated embryos did not receive any further treatment;

(2) Alpha-particle-irradiated and DU-dosed group (IU): in which the dechorionated embryos received an alpha-particle dose of $0.44 \mathrm{mGy}$ at $5 \mathrm{hpf}$ and were then exposed to $100 \mu \mathrm{g}^{-1}$ of DU for $1 \mathrm{~h}$ (from 5 to $6 \mathrm{hpf}$ );

(3) Irradiated group (I): in which the dechorionated embryos received an alpha-particle dose of 0.44 $\mathrm{mGy}$ at $5 \mathrm{hpf}$;
(4) DU-dosed group (U): in which the dechorionated embryos were exposed to $100 \mu \mathrm{g} \mathrm{l}^{-1}$ of DU for $1 \mathrm{~h}$ (from 5 to $6 \mathrm{hpf}$ ).

The experimental flow involving embryos in these four groups is schematically shown in Figure 1. The experiments were repeated three times. As described earlier, 40 dechorionated embryos were employed for each replicate. In each Petri dish, $3 \mathrm{ml}$ of medium was used. The embryos in Groups I and $U$ were exposed to alpha particles and DU, respectively, whereas those in Group IU were exposed first to alpha particles and then to DU.

\section{Alpha-particle irradiation}

Alpha-particle irradiation of zebrafish embryos was performed with the set-up described by Yum et al. ${ }^{(7)}$ The irradiation dish was a Petri dish with a 35-mmdiameter hole at the centre covered by a $3.5-\mu \mathrm{m}$-thick biocompatible Mylar film (Dupont, Hong Kong) substrate, which was fixed to the dish using an epoxy (Araldite Rapid, England). An ${ }^{241}$ Am source emitting alpha particles with an energy of $5.49 \mathrm{MeV}$ under vacuum and an activity of $4.26 \mathrm{kBq}$ was employed.

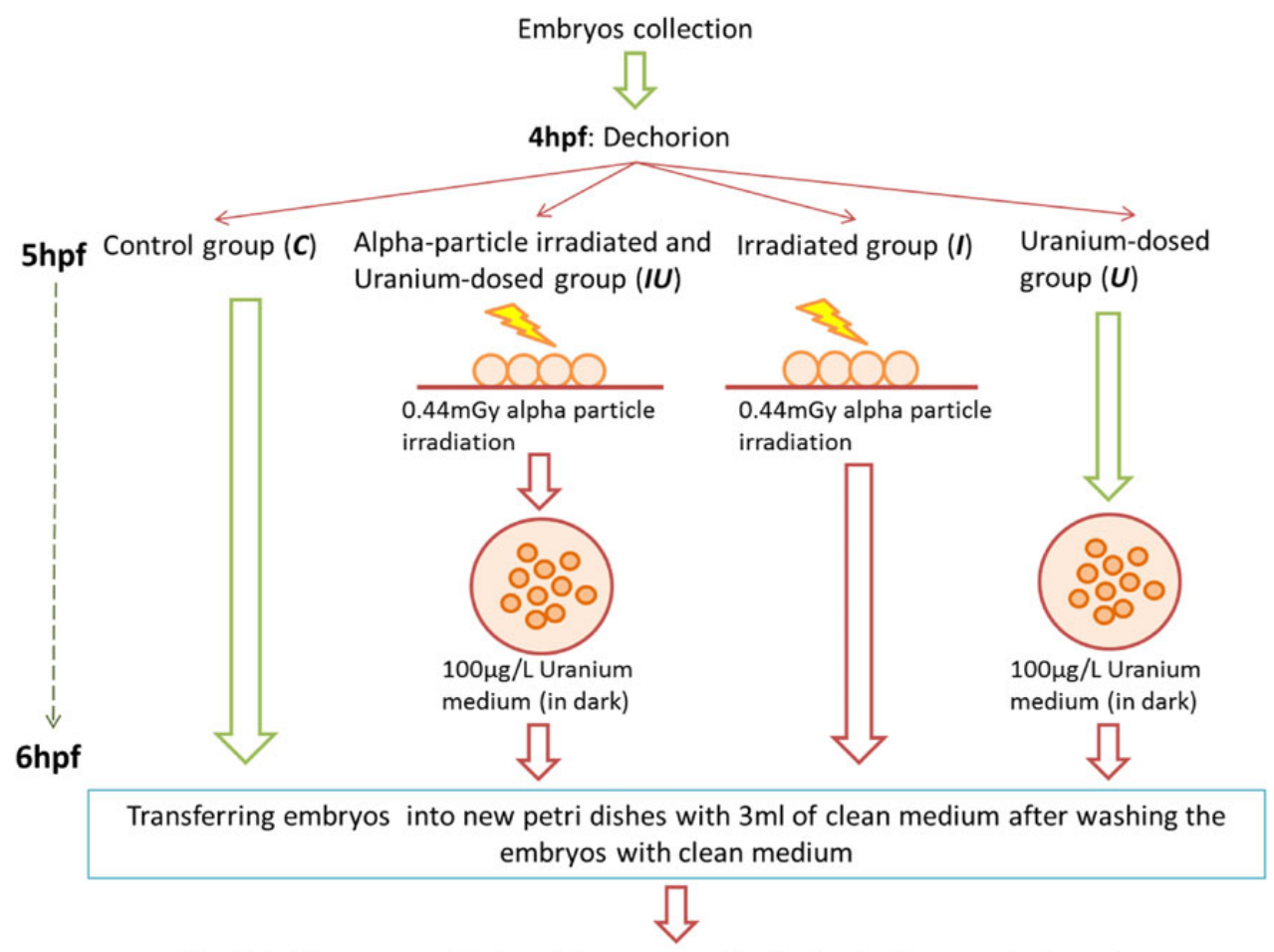

24hpf: Acidine orange (AO) staining to quantify the level of apoptosis in embryos

Figure 1. The experimental flow involving embryos in the control group (C), alpha-particle irradiated and DU-dosed group (IU), irradiated group (I) and DU-dosed group (U). 
At $5 \mathrm{hpf}$, the embryos in Groups I and IU were transferred onto the substrate in the irradiation dish and irradiated with alpha particles for $24 \mathrm{~s}$, which corresponded to an absorbed dose of $\sim 0.44 \mathrm{mGy}$. Yum et $a l^{(8)}$ observed radiation hormesis for apoptosis when the zebrafish embryos received the same alphaparticle dose. All embryos were orientated in such a way that the cells faced down towards the Mylar film, and the alpha particles came from below through the Mylar film instead of coming from above to avoid the problem of having different travelling distances of alpha particles in the medium before reaching the embryos. Group U also went through these procedures but was only sham-irradiated, i.e. without the use of the ${ }^{241} \mathrm{Am}$ source. After irradiation or sham-irradiation for the Groups IU and U, respectively, the embryos were then exposed to DU.

\section{Depleted uranium exposure}

In the present project, DU exposure was provided by uranyl acetate [UA, $\mathrm{UO}_{2}\left(\mathrm{CH}_{3} \mathrm{COO}\right)_{2} \bullet 2 \mathrm{H}_{2} \mathrm{O}$ ] (Electron Microscopy Sciences). For each set of experiments, a new DU stock solution was prepared to avoid the fluctuation in the DU concentration due to precipitation. To ensure UA was completely dissolved, preparation of the stock solution started $1 \mathrm{~d}$ before performing each set of experiments. The UA stock solution with a DU concentration $0.15-0.30 \mathrm{~g} \mathrm{l}^{-1}$ was prepared by dissolving UA with MilliQ water. Since UA was sensitive to light and would precipitate if exposed, all stock solution was kept at $4{ }^{\circ} \mathrm{C}$ and in dark. On the day of the experiment, the stock solution was further diluted to the desired concentration of $100 \mu \mathrm{g}^{-1}$ of DU. A volume of $3 \mathrm{ml}$ of UA working solution, which was sufficient to cover all embryos in each dish, was prepared in two new Petri dishes. Immediately after the $I U$ and $U$ groups of embryos were irradiated and sham-irradiated with alpha particles, respectively, they were removed from the original medium and transferred into the UA solution carefully with a glass dropper to provide the DU exposure. The embryos were kept in the UA solution for $1 \mathrm{~h}$ in dark. After $1 \mathrm{~h}$, the embryos were removed from the UA solution, washed with $6 \mathrm{ml}$ of clean medium and finally transferred to new Petri dishes with $3 \mathrm{ml}$ of clean medium. All four groups of embryos (C, IU, I and $\mathrm{U}$ ) were then returned to the $28^{\circ} \mathrm{C}$ incubator for further development.

\section{Quantification of apoptosis by vital dye staining}

In the present project, the biological endpoint was the number of apoptotic signals on the embryos at $24 \mathrm{hpf}$, which had been widely adopted for studying radiation effects on zebrafish embryos ${ }^{(9,10)}$. The staining procedure was described by Choi et al. ${ }^{(11)}$ The four groups of embryos were transferred into a medium containing $2 \mu \mathrm{g} \mathrm{ml}^{-1}$ of the vital dye acridine orange (AO) (Sigma, St. Louis, MO, USA), which was commonly used to quantify the level of apoptosis in zebrafish embryos ${ }^{(15-17)}$, to stain in the dark for $60 \mathrm{~min}$ and then thoroughly washed twice in the culture medium. After anaesthetizing the embryos by $0.0016 \mathrm{M}$ tricaine (Sigma), three images for each embryo focusing on different sections were captured under the fluorescent microscope with a magnification of $40 \times$, which were then combined into a single image for quantification of apoptosis signals with the help of a computer program 'Particle Counting 2.0' (developed by J. Zhang).

\section{Statistical analysis}

A total of 3 sets of experiments each with 40 zebrafish embryos had been carried out on different days. The number of apoptotic signals on each zebrafish embryo was quantified as described earlier. For each group of data, values lying outside the range of 1.5 times the interquartile range above the 75 th percentile and below the 25th percentile were classified as 'outliers', where the interquartile range was the difference between the 25th and 75th percentiles of the data. After excluding the outliers, if any, $t$-tests were used to ascertain the statistical significance of differences between samples. Cases with $p \leq 0.05$ corresponded to statistically significant differences between the compared groups.

\section{RESULTS}

\section{Effect of DU exposure}

The authors denoted the mean number of apoptotic signals for the $\mathrm{C}$ and $\mathrm{U}$ groups as $N_{\mathrm{C}}$ and $N_{\mathrm{U}}$, respectively. The results of the three sets of experiments $(N \pm \mathrm{SE})$ were shown in Table 1 , where SE was the standard error of the mean. The amount of apoptotic signals of Group $\mathrm{U}$ was significantly larger than that of Group C in all three sets of experiments. As such, exposing zebrafish embryos to a high concentration of DU $\left(100 \mu \mathrm{g}^{-1}\right)$ from 5 to $6 \mathrm{hpf}$ resulted in a toxic effect, which was reflected by the increase in the number of apoptotic signals in the embryos.

Table 1. Mean numbers of apoptotic signals $(N \pm \mathrm{SE})$ for embryos in Groups $C$ and $U$ in three sets of experiments.

\begin{tabular}{llll}
\hline Set & $N_{\mathrm{C}}$ & $N_{\mathrm{U}}$ & $p^{1}$ \\
\hline 1 & $102 \pm 9$ & $120 \pm 5$ & $0.0489^{*}$ \\
2 & $131 \pm 12$ & $164 \pm 13$ & $0.0374^{*}$ \\
3 & $114 \pm 15$ & $147 \pm 10$ & $0.0456^{*}$ \\
\hline
\end{tabular}

\footnotetext{
${ }^{1} p$-values from comparing Groups $\mathrm{C}$ and $\mathrm{U}$ using $t$-tests.

${ }^{*}$ Cases with $p \leq 0.05$ are considered statistically significant.
} 


\section{Y. P. NGET AL.}

Table 2. Mean normalised net numbers of apoptotic signals $(N \pm \mathrm{SE})$ for Groups $\mathrm{C}, \mathrm{IU}, \mathrm{I}$ and $\mathrm{U}$ in three sets of experiments. Total number of embryos $=117$ in all groups after removing outliers, if any.

\begin{tabular}{lcc}
\hline$N_{\mathrm{IU}}{ }^{*}$ & $N_{\mathrm{I}}{ }^{*}$ & $N_{\mathrm{U}}{ }^{*}$ \\
\hline $0.409 \pm 0.081$ & $\begin{array}{l}-0.163 \pm 0.047 \\
p=1.26 \times 10^{-7^{*}, \mathrm{a}}\end{array}$ & $\begin{array}{l}0.240 \pm 0.049 \\
p=0.0396^{*}, \mathrm{~b}\end{array}$ \\
\hline
\end{tabular}

${ }^{\mathrm{a}} p$-values obtained by comparing Groups IU and I using $t$-test.

${ }^{\mathrm{b}} p$-values obtained by comparing Groups IU and U using $t$-test.

*Cases with $p \leq 0.05$ are considered statistically significant.

\section{Multiple stressor effect of alpha-particle irradiation and DU exposure}

If $N_{\mathrm{C}}$ was taken as the average number of background apoptotic signals for embryos in the corresponding set of experiments, the net apoptotic signals for Groups IU, I and U could be described as $N_{\mathrm{IU}}{ }^{\mathrm{Net}}=\left(N_{\mathrm{IU}}-N_{\mathrm{C}}\right)$, $N_{\mathrm{I}}^{\text {Net }}=\left(N_{\mathrm{I}}-N_{\mathrm{C}}\right)$ and $N_{\mathrm{U}}^{\mathrm{Net}}=\left(N_{\mathrm{U}}-N_{\mathrm{C}}\right)$, respectively. Thus, the normalised net apoptotic signal (similar to the excessive relative risk) for these groups could be expressed as $N_{\mathrm{IU}}{ }^{*}=\left[N_{\mathrm{IU}}{ }^{\mathrm{Net}} / N_{\mathrm{C}}\right], N_{\mathrm{I}}^{*}=\left[N_{\mathrm{I}}^{\mathrm{Net}} / N_{\mathrm{C}}\right]$ and $N_{\mathrm{U}}{ }^{*}=\left[N_{\mathrm{U}}^{\mathrm{Net}} / N_{\mathrm{C}}\right]$. The experiments were repeated three times, and the normalised net data were grouped for analyses. The results were shown in Table 2. The presence of radiation hormesis in zebrafish embryos having received a low alpha-particle dose ( $\sim 0.44 \mathrm{mGy}$ ) was confirmed by the negative normalised net apoptotic signal for Group I. Surprisingly, when alpha-particle irradiation was supplemented by further exposure to $100 \mu \mathrm{g}^{-1}$ of DU (Group IU), the amount of apoptosis on the embryos became larger than that for embryos in Group U.

\section{DISCUSSION}

The results reported in the present paper were the first to demonstrate that the beneficial effect (hormesis on apoptotic signals) brought about by one stressor (alpha-particle irradiation) could be changed by the simultaneous presence of another stressor (DU) into an apparently toxic effect. Interestingly, this combined effect could hardly fall into any well-known categories of multiple stressor effects including additive, synergistic or antagonistic effects, which were defined for toxic effects of the individual stressors.

It is remarked here that, by taking into account the detailed information such as the specific activity of DU, ranges of alpha particles in water, the volume of the sensitive cells in 5-hpf zebrafish embryos etc., the radiation dose derived from DU exposure received by the $I U$ group of embryos was found to be many orders of magnitude lower than the radiation dose derived from alpha particles emitted from the ${ }^{241} \mathrm{Am}$ source. As such, perturbation from DU exposure to the alpha-particle dose from the ${ }^{241} \mathrm{Am}$ source could be safely neglected.

Explanation of the multiple stressor effect obtained in the present work would require understanding on the patterns of and mechanisms behind the cell deaths induced by alpha-particle irradiation (in the hormetic zone) and by DU (in the toxic zone). Hoffmann ${ }^{(3)}$ summarised various mechanisms contributing to hormetic responses at low doses, whereas Calabrese $^{(4)}$ gave a comprehensive review on mechanisms of hormetic dose/concentration responses with focus on those mediated via receptor and/or cell signalling pathways. The multiple stressor effect obtained in the present work could be explained in terms of the promotion of early death of cells predisposed to spontaneous transformation ${ }^{(3)}$ by the small alpha-particle dose (i.e. hormetic effect) and the postponement of cell death upon DU exposure ${ }^{(5)}$. Of course, a clearer picture could be provided by further studies on different combinations of alpha-particle dose and DU exposure, e.g. (high alpha-radiation dose + low DU exposure), (high alpha-radiation dose + high DU exposure) and (low alpha-radiation dose + low DU exposure), which would be carried out in the authors' future studies.

\section{FUNDING}

The present work was supported by the PROCOREFrance/Hong Kong Joint Research Scheme, funded by the Research Grants Council and the Consulate General of France in Hong Kong [CityU Grant number: 9052012].

\section{REFERENCES}

1. Hertzberg, R. C. and Teuschler, L. K. Evaluating quantitative formulas for dose-response assessment of chemical mixtures. Environ. Health Perspect. 110, 965-970 (2002).

2. US EPA. Framework for Cumulative Risk Assessment. EPA/630/P-02/001F. US Environmental Protection Agency (2003).

3. Hoffmann, G. R. A perspective on the scientific, philosophical, and policy dimensions of hormesis. Dose Response 7, $1-51$ (2009).

4. Calabrese, E. J. Hormetic mechanisms. Crit. Rev. Toxicol. 43, 580-606 (2013).

5. Miller, A. C., Brooks, K., Stewart, M., Anderson, B., Shi, L., McClain, D. and Page, N. Genomic instability in human osteoblast cells after exposure to depleted uranium: delayed lethality and micronuclei formation. J. Environ. Radioact. 64, 247-259 (2003).

6. Barbazuk, W. B., Korf, I., Kadavi, C., Heyen, J., Tate, S., Wun, E., Bedell, J. A., McPherson, J. D. and 


\section{DEPLETED URANIUM AND IONISING RADIATION}

Johnson, S. L. The syntenic relationship of the zebrafish and human genomes. Genome Res. 10, 1351-1358 (2000).

7. Yum, E. H. W., Ng, C. K. M., Lin, A. C. C., Cheng, $\mathrm{S}$. H. and $\mathrm{Yu}, \mathrm{K}$. N. Experimental setup for studying the effects of alpha particles on zebrafish embryos. Nucl. Instrum. Meth. B 264, 171-176 (2007).

8. Yum, E. H. W., Li, V. W. T., Choi, V. W. Y., Cheng, S. H. and $\mathrm{Yu}, \mathrm{K}$. N. Effects of alpha particles on zebrafish embryos. Appl. Radiat. Iso. 68, 714-717 (2010).

9. Geiger, G. A., Parker, S. E., Beothy, A. P., Tucker, J. A., Mullins, M. C. and Kao, G. D. Zebrafish as a 'Biosensor'? Effects of ionizing radiation and amifostine on embryonic viability and development. Cancer Res. 66, 8172-8181 (2006).

10. Bladen, C. L., Lam, W. K., Dynan, W. S. and Kozlowski, D. J. DNA damage response and Ku80 function in the vertebrate embryo. Nucl. Acids Res. 33, 3002-3010 (2005).

11. Choi, V. W. Y., Lam, R. K. K., Chong, E. Y. W., Cheng, S. H. and Yu, K. N. Designing experimental setup and procedures for studying alpha-particle-induced adaptive response in zebrafish embryos in vivo. Nucl. Instrum. Meth. B 268, 651-656 (2010).
12. Yu, K. N., Tung, M. M. T., Choi, V. W. Y. and Cheng, S. $\mathrm{H}$. Alpha radiation exposure decreases apoptotic cells in zebrafish embryos subsequently exposed to the chemical stressor, Cd. Environ. Sci. Pollut. Res. 19, 3831-3839 (2012).

13. Choi, V. W. Y., Ng, C. Y. P., Kong, M. K. Y., Cheng, $\mathrm{S}$. H. and $\mathrm{Yu}, \mathrm{K}$. N. Adaptive response to ionizing radiation induced by cadmium in zebrafish embryos. J. Radiol. Prot. 33, 101-112 (2013).

14. Ng, C. Y. P., Choi, V. W. Y., Lam, A. C. L., Cheng, S. H. and $\mathrm{Yu}, \mathrm{K} . \mathrm{N}$. Multiple stressor effect in zebrafish embryos from simultaneous exposures to ionizing radiation and cadmium. J. Radiol. Prot. 33, 113-121 (2013).

15. Yasuda, T., Yoshimoto, M., Maeda, K., Matsumoto, A., Maruyama, K. and Ishikawa, Y. Rapid and simple method for quantitative evaluation of neurocytotoxic effects of radiation on developing Medaka brain. J. Radiat. Res. 49, 533-540 (2008).

16. Tucker, B. and Lardelli, M. A. Rapid apoptosis assay measuring relative acridine orange fluorescence in zebrafish embryos. Zebrafish 4, 113-116 (2007).

17. Mei, J., Zhang, Q. Y., Li, Z., Lin, S. and Gui, J. F. Clqlike inhibits p53-mediated apoptosis and controls normal hematopoiesis during zebrafish embryogenesis. Develop. Biol. 319, 273-284 (2008). 